\title{
Lichtreaktionen der 0xyde des Titans, Cers und der Erdsäuren
}

von

Carl Renz.

(12. X. 21.)

Titandioxyd, Cerdioxyd ${ }^{\mathbf{1}}$, Niobpentoxyd und Tantalpentoxyd sind an sich lichtbeständige Körper, erlangen aber in geeignetem Mediun teilweise eine sehr erhebliche Lichtempfindlichkeit.

Bei diesen Lichtreaktionen handelt es sich um Reduktionen. Die Wirkung des Lichts ist hier umso augenfälliger, als die betreffenden Oxyde bis jetzt nur in reduzierender Atmosphäre bei hohen Temperaturen in niedrigere Oxydationsstufen übergeführt werden konnten.

\section{Titandioxyd und Cerdioxyd.}

a) Titandioxyd.

Weisses Titandioxyd, $\mathrm{TiO}_{2}$, wird unter Glycerin ${ }^{2}$ ) bei der Insolation an der Belichtungsfläche rasch blaugrau und dann dunkelblaugrau. Es ist hierzu nicht einmal direktes Sonnenlicht nötig, es liegt also eine sehr sensible Lichtreaktion vor.

Am belichteten Bodenkörper bilden sich nach einiger Zeit Gasbläschen, jedenfalls Kohlendioxyd. Bei längerem Stehen im Dunkeln blasst die Färbung wieder etwas ab.

Die gleiche Erscheinung zeigt sich unter Glycerin mit einer Beigabe von Kaliumhydroxyd, während ein Salzsäurezusatz (Chlorhydrine) zunächst den sichtbaren Lichteffekt verringert. In letzterem Falle geht aber Titan infolge der Reduktion als Titantrichlorid in Lösung und die Flüssigkeit färbt sich lila. Nach längerer Belichtung fällt Ammoniak aus der violetten Lösung schwarzes Titanihydroxyd $\left(\mathrm{Ti}(\mathrm{OH})_{3}\right)$.

1) Hinsichtlich des Cerdioxyds verweise ich auch auf Carl Renz, Lichtreaktionen mit Cerdioxyd. Z. an. Ch. 110, 104-106 (1920).

2) Glycerin geht im Licht an sich in Glycerose (Dioxyaceton) iiber. 
Unter reiner Salzsäure (38\%) tritt ülerhaupt keine oder anfangs höchstens eine ganz leichte Graufärbung de: Bodenkürpers ein, die Salzsäure wird jedoch gelb. Nach längerer Exposition fällt Ammoniak aus ler Salzsäure reichlich Titansäure. Unter der ausfallenden Titansäure bleibt am Boden des Reagierglases eine bräunlichlila Flïssigkeitsschicht, aus der sich noch etwas dunkles Titanihydroxyl alscheidet, überdeckt von der Hauptmasse der Titansäure. Kalilauge fällt Titansäure von Graulilaton. In der Salzsäure ist daher Titan im drei- und vierwertigen Zustand, und zwar z. T. als Titanchlorwasserstoffuäure enthalten, indem sich das in reduziertem Stadium gelöst. Titan grö̈stenteils wieder oxydiert.

Ebenso wie unter Glycerin färbt sich 'Jitandioxyd in Licht unter konz. wässrigen Lösungen von Mannit, Querzit. und Traubenzucker; besonders unter Mannit- und l'raubenzuckerlösungen setzt die intensive Blaugraufärbung alsbald ein.

Ferner eignen sich zur Hervorrufung dir lichtreduktion abs. alkoholische Lösungen von Weinsäure, Zitronensäure oder Mandelsäure, unter denen das Titandioxyd an der Belichtungsstelle sogleich blaugrau zu werden beginnt. Namentlich unter alkoholiseher Mandelsäure und Zitronensäure wird die dunkelhlangrane Endfürbung, wie unter Glycerin, erreicht. Über Nacht stellt sich jeweils von ohen her eine mehr oder minder starke Aufhellung (Oxydation) ein, besonders unter alkoholischer Zitronensäure. Unter wiissrigen Woinsäurelösungen tritt die Färbung weniger kräftig hervor und geht unter Bildung von Gasperlen (Kohlendioxyd) nur bis blangrau, dewgleichen unter alkoholischer Opiansäure. Protocatechusäurelösungen sind unhrauchbar, sie werden mit Titandioxyd schon im Dunkeln orangerot.

In geringerem Grade verändert sich Titandioxỵd unter flüssigen Kohlenwasserstoffen, z. B. unter Xylol (blaugrau, im I)unkeln Rückbildung), sowie unter Benzaldehyd (balcl, aber nur blaugrau), noch schwächer unter Cuminol (leicht blaugrau), Zimtaldehyd und Furfurol (nur Grauschimmer) und gar nicht unter alkoholischem Vanillin. Stannochloridlösung wirkt im Licht ebenfalls reduzierend. Versuche mit Pyridin verliefen resultatlos, dagegen bewähren sich manche Alkalö̈de, wie Atropin (in alkoholischer Lösung), wiederum als ausgezeichnete Förderungsmittel der Lìhtreduktion. 'Titandioxyd färbt sich unter alkoholischem Atropin, sellst bei bedecktem Ilimmel, rasch blaugrau und in der Sonne dunkellblaugrau, die rom Licht abgekehrte Rückseite bleibt weiss (im Dunkıln von obenher Rückgang der Fürbung). 
Lnter Phenylhydrazin wird das Titandioxyd an der besonnten Stelle schnell blaugrau und dunkelt bei längerer Bestrahlung weiter nach, über Nacht verfärbt es sich jeweils wieder. Die Sonnenwärme spielt bei der Reduktion kaum mit, da das Phenylhydrazin in der Hitze unter leichter Grautönung nur wenig auf Titandioxyd einwirkt.

Vom Titan existieren ausser dem Dioxyd noch ein schwarzes Titanmonoxyd, TiO, das in reinem Zustand zurzeit nicht mit Sicherheit bekannt ist, und ein ebenfalls schwarzes 'Titansesquioxyd, $\mathrm{Ti}_{2} \mathrm{O}_{3}$.

Entsprechend dem Titandioxyd verhalten sich seine Hydrate (Titansäure) gegenüber der Iichtwirkung.

Titansäure wird, unter Glycerin exponiert, binnen kurzem blaugrau, dunkelblaugrau und blauschwarz. Unter Mannit- und Trauhenzuckerlösungen entwickelt sich gleicherweise die blaugraue und unter Mannit auch dunkelblaugraue Färbung. Dureh ans $h$ hliessende Dunkelreaktion erfolgt ebenfalls eine Aufhellung.

Trocken belichtet bleibt die Titansäure, wie ihr Anhydrid, unverändert. Allerdings handelt es sich in beiden Fällen wohl nur um eine scheinbare Unempfindlichkeit, die Wirkung kommt eben wegen der unmittelbaren Reoxydation nicht zum Ausdruck.

\section{b) Cerdioxyd.}

Cerdioxyd, $\mathrm{CeO}_{2}$, färbt sich unter Glycerin nach kürzerer Belichtung grau und graulila - je nach der Herkunft bald heller, bald dunkler - und bleibt dann bei längerer Besonnung stationär. Unter Mannitlösung ist der Lichteffekt noch geringer.

Am schnellsten und kräftigsten verändert sich das Cerdioxyd unter Benzaldehyd, unter dem an der belichteten Fläche in kurzer Zeit die Graulilafärbung erscheint. Dasselbe, wenn auch etwas schwächere Ergebnis veranlasst Zimtaldehyd, während Cuminol sich als noch weniger wirksam erwcist.

Mit Phenylhydrazin tritt auch in der Hitze Graufärbung ein, die sich dann im Licht noch verstärkt.

Bei der Belichtung des Cerdioxyds unter Salzsäure ( $38 \%$ ) bemerkt man gleichfalls einen Grauschimmer; am bestrahlten Teil des Bodenkörpers bilden sich Gasbläschen, jedenfalls Sauerstoff, während die Salzsäure gelb wird. Nach einiger Zeit hört die Bläschenentwicklung auf; die Salzsäure blasst im Dunkeln wieder ab. Kalilauge fällt aus der Salzsäure Cerohydroxyd, Ammoniak ebenfalls; bisweilen zeigt sich hier, wie beim Titandioxyd, zunächst unter dem ausfallenden Cerohydroxyd eine bräunlichlila Flüssigkeitsschicht.

Es sind daher in der Salzsäure neben Cero- auch in geringem Grade Ceriionen vorhanden infolge Oxydation des im Licht gelösten Cerochlorids. 
II. Erdsäureanhydride.

Analog dem Titandioxyd und Cerdioxyd reagiert das Pentoxy des Niobs (Niobsäureanhydrid) im Sonnenliclıt bei Gegenwart verschiedener organischer Medien.

Weisses Niobpentoxyd, $\mathrm{Nb}_{2} \mathrm{O}_{5}$, färbt sich unter Glycerin nach kurzer Exposition zunächst grau, dann dun kelgra ubla und schliesslich tief schwarzblau (stahlblau); es wird hier also unter gleichen Vorbedingungen eine weit intensivere Färbung erreicht, als beim Cerdioxyd und auch beim Titandioxyd. Die Farbenänderung, die im Dunkeln andauert, beschränkt sich ausschliesslich auf den rom Licht getroffenen Teil des Bodenkörpers. Der Lichteffekt erfolgt auch unter Glycerin nit einem Zusatz von Kaliumhydroxyd orler Salzäure.

Unter konz. wässrigen Lösungen ron Mannit, Querzit und Traubenzucker beginnt an der Belichtungsfläche ebenfalls bald die Grautönung, die dann zu dunkelgraublau und schwarzblau weiterschreitet, besonders ausgesprochen unter Vannitlïsung, weniger unter Traubenzucker. Die obere Berührungsschicht des Bodenkörpers mit der Flüssigkeit bleibt hierbei anfangs heller, über Nacht geht jeweils auch die Färbung der Grundmasse von oben narh unten zurück, jedenfalls weil der Luftsauerstoff von diesen Flüssigkeiten weniger abgehalten wird, wie von Glycerin.

In gleicher Art fördern eine Reihe rrganischer Säuren in abs. alkoholischer Lösung die Lichtreaktion. Das Niobpentoxyd wird unter alkoholischen Lösungen von Zitronensäure, Wreinsäu re und Ma ndelsäure nach kürzerer Belichtung grau, dunkelgraublau und schwarzblau, und zwar nur einseitig an der Insolationsfläche. Der obere Rand des Bodenkörpers färbt sich hierbei zuletzt, ehen*o geht die Färbung von oben her über Nacht jeweils mehr oder ninder zurück. I'nter alkoholischer Zitronensäure wurde eine besonders tief schwarze Endfärbung mit Blaureflex erzielt, unter alkoholischer Nandel*äure hebt sich die blaue Nuancierung schärfer hervor.

Unter wärsriger Weinsäurelösung wird die Täarbung weniger kräftig und mehr grau, auch erfolgt hier im Dunkeln eine stärkere Aufhellung. Das Gleiche gilt für die Bestrahlung unter Benzaldehyd (grau und dunkelgraublau), Zimtaldehyd (nur lichtgraublau), (uminol, Furfurol (unter den beiden letzteren Flüssigkeiten nur Graufärbung), sowie unter Phenylhydrazin. Beim Erhitzen mit Phenylhydrazin wird das Niobpentoxyd nur leicht grau, es dunkelt dann im Sonnenlicht sofort entsprechend nach; die Rückseite bleiht jedoch hiervon unberiihrt, 
das Licht leistet daher auch hier die Hauptarbeit bei der Reduktion. Pyridin und alkoholisches Vanillin sind, wie beim Titandioxyd, wirkungslos, ebenso alkoholische Atropinlösung, letztere jedoch im Gegensatz zum Titandioxyd.

Unter Salzsäure (38\%) belichtet, erfährt das Niobpentoxyd keine sichtbare Änderung. Die Salzsäure färbt sich nach und nach gelb; Ammoniak und Alkalien fällen hieraus weisse Niobsäure, und zwar nach längerer Besonnung in relativ reichlicher Menge. Die Löslichkeit wird auch in diesem Falle durch die Lichtreduktion bedingt; in der Salzsäure ist jedenfalls infolge Oxydation der sich bildenden Chlorverbindung Niobpentachlorid oder auch_Nioboxychlorid enthalten. Je nach Lmständen färbt sich die Salzsäure bezw. der Niederschlag bei der Neutralisation mit Ammoniak auch braunlila, ein Zeichen, dass sie noch niedrigervalentes Niob enthält.

Ähnlich ihrem Anhydrid erweist sich auch die Niobsäure als lichtempfindlich, nur neigt der Farbenton mehr zu lilabraun (unter (alycerin).

Im Gegensatz hierzu zeigt sich das Tantalpentoxyd, $\mathrm{Ta}_{2} \mathrm{O}_{5}$, unverhältnismässig weniger empfindlich.

Unter Glycerin geht die weisse Farbe dieses Erdsäureanhydrids erst nach längerer Belichtung allmählich in leichtes Grau über, ebenso unter Phenylhydrazin.

Niobhaltiges Tantalsäureanhydrid reagiert dagegen wie Niobpentoxyd, wenn auch nicht das ganz tief schwarzblaue Endresultat erreicht wird.

Da sich die beiden in der Regel zusammen vorkommenden Erdsäuren nur schwierig vollständig von einander trennen lassen, dürfte das Tantalpentoxyd überhaupt im Licht unveränderlich sein und die beobachtete Grautönung an sonst reinem Tantalpentoxyd durch Niobspuren verursacht werden. Die Lichtreaktion wäre sonach ein zweckdienliches Mittel, um die beiden Erdsäuren zu unterscheiden.

Das Niobpentoxyd lässt sich auch auf chemischem Wege weitgehender reduzieren, als das Tantalpentoxyd; es bildet zwei, vielleicht sogar drei niedrigere Oxydationsstufen: das Niobtetroxyd, $\mathrm{Nb}_{2} \mathrm{O}_{4}$, ein schwarzes Pulver mit bläulichen Reflexen, und das bläulichschwarze Niobtrioxyd, $\mathrm{Nb}_{2} \mathrm{O}_{3}$, während man vom Tantal, abgesehen vom Pentoxyd, nur ein dunkles Tantaltetroxyd, $\mathrm{Ta}_{2} \mathrm{O}_{4}$ kennt.

Die Lichtreduktion führt daher beim Niobpentoxyd jedenfalls zunächst zum Niobtetroxyd, wenn auch unter besonders wirksamen Medien (wie Glycerin, alkoholischer Zitronensäure) eine noch weitergehende Reduktion bis zum Niobtrioxyd stattfinden könnte. 
III. Ergebnisse und Vergleiche.

Meine Lichtversuche haben daher erweben, dass vor allem das Titandioxyd und Niobpentoxyd, aber auch das Cerdioxyd unter verschiedenen organischen Flüssigkeiten und reduzierenden Lösungen eine \%. T. sehr beträchtliche Lichtempfindlichkeit besitzen, indem sie zu gefärbten, niedrigeren Oxydationsprodukten reduziert werden.

Am besten eignet sich für diese Lichtreduktionen im allgemeinen las Glycerin, das eine Reoxydation zurückhält, indem es den Zutritt ler Luft erschwert. Dass es sich tatsächlich un Reduktionserscheinungen handelt, geht daraus hervor, dass sich dir nach der Belichtung ausgewaschenen ()xyde meist schon beim Trocknen an der Luft aufhellen und beim Erhitzen wieder die ursprünglichen I loxyde hezw. Pentoxyde liefern. Ebenso tritt auch unter den meisten der benuitzten Stoffe schon im I)unkeln wicder eine Rückbildung ein.

Es ist jedoch nicht anzunehmen, dass lie Photoreduktion bis zum Vetall führt, da die entsprechenden Lichtreaktionen bei dem Zwischenglied der Titan-Cerreihe, dem Zirkonium, dat keine niedrigeren Oxyde bildet, ausbleiben.

Dagegen kämen beim Niobpentoxyd akmessorische Bestandteile als laktor des photochemischen Prozesses in Betracht, und zwar in erster Linie Beimengungen von Zinnsäure, Wolframsäure, Zirkonerde und Titansäure bezw. ihrer Anhydride. Bekanntlich ist die vollständlige Trennung der Erdsäuren hiervon, namentlich rom 'Titan, nur schwer zu erreichen.

Zinndioxyd wird nach längerer Belichtung unter Glycerin nur leicht grau (unter Mannitlösung noch weniver ausgesprochen) und erleirlet somit nur eine geringe Reduktion. Jie Lichtempfindlichkeit des Zinndioxyds ist daher weitaus schwächer, als die des Niohpentoxyds.

Wolframsäure (wasserfrei) wechselt im Somnenlicht unter Glycerin (henfalls ihre Farbe und geht in grünstichiges Blau aber, heibt aber auch nach langer Insolation immer wesentlich heller, als ein gleicherweise belichtetes Niobpentoxyd. Trocken hestrahlt ist die Wolframsäure keiner Farbenänderung unterworfen; mit Phenylhydrazin erhitzt geht sie unter lebhafter Reaktion in blau und schwarz über, hellt sich aher beim Stehen, auch im Licht, zu dunkelblau mit Grunstich auf.

Zirkoniumdioxyd ändert sich bei der Besomnung unter Glycerin und beim Belichten oder Erhitzen mit Ph'nylhydrazin in keiner bemerkbaren Art. Eine möglicherweise ganz sihwache Graufärhung nach längerer Exposition kann hier ansser Betracht fallen. 
Es bleibt also noch das Titandioxyd, dessen Lichtreaktionen und die dabei hervorgetretenen Lnterschiede gegenüber dem Niobpentoxyd in den vorstehenden Abschnitten beschrieben wurden.

Die Färbungsnüance des Niobpentoxyds ist aber immer mehr grau und die Schlussfärbung, die mit der Farbe der auf chemischem Wege erhaltenen niedrigeren Nioboxyde übereinstimmt, wesentlich dunkler.

In Anbetracht der Stärke der Lichtwirkung und der Tiefe des Farbentones handelt es sich daher beim Niobpentoxyd zweifellos um eine Eigenreduktion.

Zum Vergleich wurde aus der Erdsäurenreihe noch das Vanadinpentoxyd (Vanadinsäureanhydrid) in den Kreis der Untersuchung einbezogen.

Das braune Vanadinpentoxyd, $\mathrm{Vd}_{2} \mathrm{O}_{5}$, dunkelt unter Glycerin und namentlich unter Benzaldehyd nach kurzer Belichtungsdauer an der dem Licht zugewandten Seite zu schwarz. Unter Zimtaldehyd und Cuminol (schwächer wie unter Zimtaldehyd) geht die Schwärzung ebenfalls vor sich, aber langsamer, ebenso unter wässriger Mannitlösung. Es findet hierbei eine Reduktion zu niederen Vanadinoxyden statt, die schwarz gefärbt sind, jedenfalls zunächst zu Vanadintetroxyd.

Beachtenswert ist noch das Verhalten des Vanadinpentoxyds im Licht unter abs. alkoholischen Lösungen von Zitronensäure, Weinsäure oder Mandelsäure. Nach kurzer Zeit entsteht vom belichteten Bodenkörper aus eine lebhafte Gasentwicklung (Kohlendioxyd), die Säurelösungen werden grün, die Weinsäure und Zitronensäure später blau.

Unter Mandelsäure schwärzt sich dagegen der Bodenkörper, unter der Zitronensäure zeigt sich eine vorübergehende Schwärzung nur während der Bildung der Gasperlen.

Vanadinpentoxyd und Vanadintetroxyd geben beim Stehen mit alkoholischer Zitronensäure auch im Dunkeln allmählich gleichgefärbte Lösungen, das Licht befördert jedoch auch hier die Reaktion.

Das Endglied der Titan-Cerreihe, das Thoriumdioxyd, ThO $\mathrm{O}_{2}$, wurde ebenfalls auf seine Lichtempfindlichkeit unter Glycerin geprüt. Es erleidet hierunter im Sonnenlicht keine nennenswerte Veränderung, auch beim Belichten oder Frhitzen mit Phenylhydrazin tritt keine merkliche Reduktion ein. Nach längerer Belichtung unter Glycerin erscheint beim Thoriumdioxyd allerdings ein leichter Graulilaschimmer; aber hier ist die Erscheinung derart schwach, dass sie eher, wie beim Tantalpentoxyd, von einer Verunreinigung herrühren dürfte.

Die Untersuchung wurde ausserdem auf die eigentlichen seltenen Erden ausgedehnt, und zwar auf diejenigen Glieder der seltenen Erden- 
gruppe, die neben dem allgemeinen dreiwertigen Oxydtypus noch höhere Oxyde liefern.

Braunes Praseodymhaltiges Neodymoxyd wurde unter Glycerin längere Zeit ohne Erfolg belichtet, desgleichen unter Phenylhydrazin. Dagegen verschwindet die Braunfärbung bein Erhitzen mit Phenylhydrazin und geht in lichtblaugrau üher. Es findet also mit dem Farbenwechsel jedenfalls eine Reduktion der dunkeln Praseodymperoxyde zu gelbem Praseodymoxyd $\left(\mathrm{Pr}_{2} \mathrm{O}_{3}\right)$ statt, sodass die lichtblaue Farbe des Neodymoxyds zum Vorschein kommt, die in den gewöhnlichen unreduzierten Gemischen, selbst wem ihr Praseodymgehalt nur gering ist, durch die ausserordentliche Färbekraft des schwarzen Praseodymperoxyds verdeckt wird.

Anhängend sei noch erwähnt, dass auch verschiedene andere, ausserhalb der behandelten Gruppen stehonde Oxyde unter den betreffenden organischen Flüssigkeiten eine hohe I ichtempfindlichkeit bekommen können.

So färbt sich z. B. Wismutoxyd, $\mathrm{Bi}_{2} \mathrm{O}_{3}$, unter Glycerin oder Benzaldehyd dem Sonnenlicht ausgesetzt, an der Belichtungsflärhe alsbald grau und tief schwarz. Auch unter alkoholiwcher Wcinsüure erfolgt die Schwärzung. Hierbei findet jedenfalls eine Reduktion zu Wismutoxydul, eventuell sogar zum Metall statt.

Antimonoxyde sind unter gleichen Verhältnissen ebenfalls lichtveränderlich. Antimontrioxyd wird unter Glycerin nach einiger Zeit graubraun und dunkelgraubraun.

Die Arbeiten wurden in der Tessiner Sommersonne in Reagiergläsern und Gefässen aus geschmolzenem Bergkrystall (Quarzglas) ausgeführt.

\section{Zusammenfassung.}

Titandioxyd, Cerdioxyd, Niobpentoxyd, sowie einige weitere untersuchte Oxyde liefern im Licht bei Gegenwart ('ines geeigneten organischen Akzeptors dunkel gefärbte Reduktionsprodukte. 